\title{
The nonrelativistic limit of Dirac-Fock codes: the role of Brillouin configurations
}

\author{
P. Indelicato,, , E. Lindroth, ${ }^{2}$ and J.P. Desclaux ${ }^{3}$ \\ 1 Laboratoire Kastler Brossel, École Normale Supérieure et Université P. et M. Curie, \\ Case 74, 4 place Jussieu, F-75252, Cedex 05, France \\ ${ }^{2}$ Atomic Physics, Fysikum, Stockholm University, S-106 91 Stockholm, Sweden \\ ${ }^{3} 15$ Chemin du Billery, 38360 Sassenage
}

(Dated: August 31, 2018)

\begin{abstract}
We solve a long standing problem with relativistic calculations done with the widely used MultiConfiguration Dirac-Fock Method (MCDF). We show, using Relativistic Many-Body Perturbation Theory (RMBPT), how even for relatively high- $Z$, relaxation or correlation causes the nonrelativistic limit of states of different total angular momentum but identical orbital angular momentum to have different energies. We show that only large scale calculations that include all single excitations, even those obeying the Brillouin's theorem have the correct limit. We reproduce very accurately recent high-precision measurements in F-like Ar, and turn then into precise test of QED. We obtain the correct non-relativistic limit not only for fine structure but also for level energies and show that RMBPT calculations are not immune to this problem.
\end{abstract}

PACS numbers: 31.30.Jv, 32.10.Fn, 31.25.Eb

Relativistic atomic structure codes, mostly MCDF packages, are now of widespread use in many sectors of physics, and the need for reliable, relativistic calculations is stronger than ever (see, e.g., [1] for examples in Astrophysics). However, the difficulties of doing reliable calculations are numerous, and still largely underestimated. For example a puzzle that was noted already twenty-two years ago 2] has never been solved, although it may lead even in very simple calculations to wrong energy values. In Ref. 2] it was shown that relativistic self-consistent field procedures do not produce, in a number of cases, the correct non-relativistic limit of zero for the fine structure splitting (FSS) when the speed of light is tuned to infinity. Ref. [2] suggested as a remedy explicit calculation of this non-relativistic offset (N.R.) and subsequent subtraction of it from the relativistic result, although no justification for the procedure was provided. Moreover this paper said nothing on how to correct individual energy levels. Here we will penetrate the origin of the non-relativistic shift using the tools of perturbation theory and advanced MCDF calculations. We use these tools to show the role of relaxation in the N.R. offset, and prove that the inclusion of specific mono-excitations in the MCDF basis removes it. We also provide justification to the subtraction procedure and show that not only the FSS need to be corrected, but also the level energy, e.g., when transitions between different shells are studied. It is also worth noting that this problem appears in the Optimized Level (OL) scheme when each level energy and wavefunction is optimized separately. This scheme is used only when the highest accuracy for correlation is required. Often the average (AL) level scheme is used, in which the same $J$-average wavefunction is used to calculate the energy of all FS component. In the AL scheme the N.R. offset does not appear, but the accuracy is much lower.
We will concentrate on the ground state configuration of a F-like ion which was used as a model system already in Ref. 2] as accurate measurements have been performed very recently [3]. With high experimental accuracy, even for $Z=18$, it is important to be aware of this problem which seriously affects the comparison with experiment on the present day level. We will further present accurate calculations of the fine structure splitting in F-like argon both with Relativistic Many-Body Perturbation Theory (RMBPT) and with the Multi-Configuration Dirac-Fock (MCDF) method. It is shown that by comparison with accurate experimental results $\underline{3}$ ] it is possible to test the calculations on self-energy and other radiative corrections in a true many-electron surrounding.

With RMBPT the fine-structure splitting in a F-like system is calculated as the binding energy difference between the $2 p_{1 / 2}$ and $2 p_{3 / 2}$ electron in the corresponding Ne-like system. The lowest order approximation of this binding energy is the negative of the orbital energy of the removed electron in the Hartree-Fock approximation. The remaining electrons are at this stage considered as frozen in their orbitals in spite of the removal of one electron. The most important correction to this first approximation is the relaxation of the electrons due to the presence of the hole. The term relaxation usually denotes the correction found by a single configuration restricted Hartree-Fock (or Dirac-Fock in the relativistic case) calculation in the presence of the hole. The non-relativistic shift has its origin already at this level and we will now concentrate on this shift and postpone the discussion of higher order corrections.

To analyze the relaxation for a one-hole state with perturbation theory it is natural to start from the closed shell system and systematically correct for the removal of one electron. Fig. 1 1 shows the contributions entering in second order. Fig. 1(a-b) show fluctuations to two 


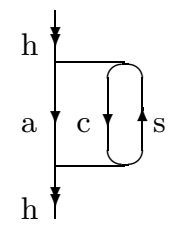

(a)

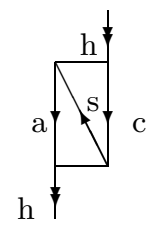

(b)

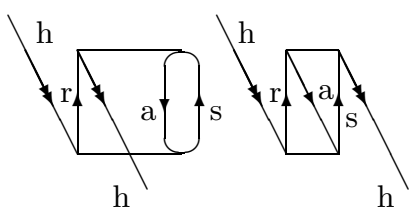

(c) (d)
FIG. 1: Illustration of the second order energy contributions to a one-hole system. Diagrams(a-b) show fluctuations to two holes and one excited orbital and diagrams (c-d) double excitations (correlation). Downgoing single arrows denote core orbitals, downgoing double arrows denote the hole and upgoing arrows denote excited orbitals.

holes and one excited orbital and Fig. 1( $($-d) true double excitations. The relaxation, i.e., the effects included by a single configuration restricted Hartree-Fock calculation is in perturbation theory part of Fig. 1(a-b); the ones where the hole is not fluctuating and the excitation from an orbital preserves its angular symmetry. The lowest order relaxation correction to an orbital $b$ can consequently be written

$$
\rho_{b}^{\text {relax }}\left(\ell_{s}=\ell_{b}, j_{s}=j_{b}\right)=-\sum_{s}^{e x c} \frac{|s\rangle\left\langle\{h s\}\left|V_{12}\right|\{h b\}\right\rangle}{\varepsilon_{b}-\varepsilon_{s}}
$$

where $h$ denotes the removed electron, the curly brackets antisymmetrization, $V_{12}$ the two-electron interaction, and the minus sign is due to the removal of $h$. The energy corrections are then calculated as

$$
\sum_{b}^{\text {core }}\left\langle\{b h\}\left|V_{12}\right|\left\{\rho_{b}^{\text {relax }} h\right\}\right\rangle .
$$

In this way all types of diagrams in Fig. 1 (a-b) with either orbital $a=h$ (and $\mathrm{b}=\mathrm{c}$ ) or $c=h$ (and $\mathrm{b}=\mathrm{a}$ ) and $\ell_{s}=\ell_{b}, j_{s}=j_{b}$ are included, i.e., the single excitations that preserve the angular structure. It can be noted that these single excitation contributions form a class of diagrams that can be summed until convergence in an iterative scheme, see, e.g., Refs. [4, [5]. Here we will not pursue this line, however, since our purpose is to analyze the relaxation in the non-relativistic limit and show why a state with a hole in $n \ell_{j=\ell-1 / 2}$ and one with a hole $n \ell_{j=\ell+1 / 2}$ do not reach the same energy in this limit. For this it is sufficient to study relaxation in second order.

As an example, take diagram Fig. 1(a) with orbital $a=h$ and $\ell_{s}=\ell_{b}$, a typical relaxation contribution. The orbitals used to evaluate the diagram are solved using the Hartree-Fock potential from the closed shell core and the radial part of the $2 p_{1 / 2}$ and the $2 p_{3 / 2}$ orbital will be identical when we let $c \rightarrow \infty$. The problem comes instead from the spin-angular part. Since

$$
\left|\ell m_{\ell} s m_{s}\right\rangle=\sum_{j m_{j}}\left|(\ell s) j m_{j}\right\rangle\left\langle(\ell s) j m_{j} \mid \ell m_{\ell} s m_{s}\right\rangle
$$

decoupling of spin and orbital angular momentum cannot be done without summing over all total angular momenta, $j$. An unambiguous way to see how this influences our example of Fig. (1) with orbital $a=h$ and $\ell_{s}=\ell_{b}$ is to compare the angular contribution non-relativistically and relativistically. The electron-electron interaction is expressed as

$$
\frac{1}{r_{12}}=\sum_{k} \frac{r_{<}^{k}}{r_{>}^{k+1}} \mathbf{C}^{k}(1) \cdot \mathbf{C}^{k}(2),
$$

where $k$ denotes the rank of the spherical tensor operator $\mathbf{C}$, which works on the orbital part of wavefunctions. Non relativistically the angular part can be evaluated as

$$
\sum_{b}^{\text {core }} \sum_{k} 2 \frac{1}{2 k+1} \frac{1}{2 \ell_{h}+1}\left\langle\ell_{h}\left\|\mathbf{C}^{k}\right\| \ell_{h}\right\rangle^{2}\left\langle\ell_{b}\left\|\mathbf{C}^{k}\right\| \ell_{b}\right\rangle^{2} .
$$

This is in fact identical to the following expression in the coupled space where two extra sums appear over intermediate total angular momenta

$$
\begin{gathered}
\sum_{b}^{\text {core }} \sum_{k} \sum_{j_{h^{\prime}}}^{\ell_{h} \pm \frac{1}{2}} \sum_{j_{b^{\prime}}}^{\ell_{b} \pm \frac{1}{2}} \frac{1}{2 k+1} \frac{1}{2 j_{h}+1} \\
\left\langle j_{h}\left\|\mathbf{C}^{k}\right\| j_{h^{\prime}}\right\rangle^{2}\left\langle j_{b}\left\|\mathbf{C}^{k}\right\| j_{b^{\prime}}\right\rangle^{2}
\end{gathered}
$$

That these two expressions give the same result can be understood by standard angular momentum algebra techniques.

In a restricted Dirac-Fock calculation there will be no sums over intermediate angular momenta. Instead only $j_{h}=j_{h^{\prime}}$ is allowed, i.e., the hole is not allowed to fluctuate to the other fine structure component, and $j_{b^{\prime}}=j_{b}$ is required, i.e., the corrections to orbital $b$ do not change its angular structure. The spin-angular part used is thus

$$
\sum_{b}^{\text {core }} \sum_{k} \frac{1}{2 k+1} \frac{1}{2 j_{h}+1}\left\langle j_{h}\left\|\mathbf{C}^{k}\right\| j_{h}\right\rangle^{2}\left\langle j_{b}\left\|\mathbf{C}^{k}\right\| j_{b}\right\rangle^{2},(7)
$$

which will clearly not produce the same result as Eq. (5), and which further cannot give identical results for, e.g., $j_{h}=1 / 2$ and $j_{h}=3 / 2$, which is easily seen from the $k=2$ contribution which is zero for $j_{h}=1 / 2$, but not for $j_{h}=3 / 2$. The difference can also be readily demonstrated numerically for a system as F-like neon where the second order contribution, Eq. 2] to the relaxation gives an unphysical fine structure offset of $0.024 \mathrm{eV}$ in the $c \longrightarrow \infty$ limit. Following the recipe from Ref. 2] and correcting the result calculated with the true value of $c$ with this offset, we obtain a relaxation contribution to the fine structure splitting of $-0.058 \mathrm{eV}$. After iteration of the relaxation contributions [4, 5] the corrected value reaches $\sim-0.050 \mathrm{eV}$, in line with the MCDF Coulomb relaxation contribution of $\sim-0.049 \mathrm{eV}$, listed in the third section of Table This value has been corrected using 
TABLE I: Summary of the contributions. All calculations use the 2002 values for fundamental constants [6, 7] $(\mathrm{eV})$. Experimental values are from wavelength provided in Ref. [3] converted to vacuum values using [8]

\begin{tabular}{lrrr}
\hline \hline & $2 p_{1 / 2}$ & $2 p_{3 / 2}$ & \multicolumn{1}{c}{$\Delta$} \\
\hline \multicolumn{4}{c}{ Contributions } \\
Ne-like DF orb. ener. & 426.50002 & 424.13211 & 2.36791 \\
$\Delta$ DF-Breit & -0.22659 & -0.13576 & -0.09083 \\
h.o. retardation & -0.00011 & 0.00079 & -0.00090 \\
QED corr. & 0.01353 & 0.00755 & 0.00598 \\
\hline \multicolumn{4}{c}{ Contributions specific to RMBPT } \\
2nd order core-core, Coul & -4.48509 & -4.42587 & -0.05921 \\
core-core, Breit & -0.01187 & -0.00814 & -0.00373 \\
correlation, Coul & 2.56726 & 2.55763 & 0.00962 \\
correlation, Breit & 0.02391 & 0.02018 & 0.00373 \\
h.o. contr. (Coul. + Breit) & 0.16559 & 0.15885 & 0.00674 \\
$\Delta$ DF Breit orbitals & 0.00198 & 0.00043 & 0.00156 \\
\hline Total (RMBPT) & 424.54863 & 422.30777 & 2.24086 \\
Experiment & & & 2.24010 \\
\hline Contributions specific to MCDF (N.R. offset subtracted) \\
Relaxation (Coul) & -3.10800 & -3.05931 & -0.04869 \\
Relaxation (Breit) & -0.00406 & -0.00314 & -0.00092 \\
Correlation (Coul $\rightarrow 5 g$ ) & 1.42466 & 1.39604 & 0.02862 \\
Correlation (Breit $\rightarrow 5 g)$ & -0.01359 & 0.00741 & -0.02100 \\
\hline Total (MCDF) & 424.58585 & 422.34569 & 2.24016 \\
Experiment & & & 2.24010 \\
\hline \hline
\end{tabular}

the same procedure. The small difference is probably due to small differences in the classification of relaxation and correlation contributions. The lesson here is that since the summation over all possible couplings of spin and orbital angular momenta of the intermediate states are necessary to reproduce the uncoupled situation a correct non-relativistic limit cannot be achieved with any single configuration self consistent field calculation. In other words, still for the system under consideration, one has to include more than one configuration relativistically to reproduce the single configuration non-relativistic result in a relativistic framework. In RMBPT the full contribution from Fig. 1(a-b) produces no N.R. offset, but any attempt to speed up the convergence of the perturbation expansion by singling out the important subclass that only involve energy denominators as in Eq. 1 will do so. The RMBPT results shown in Table凹are obtained without any such procedure and has a correct non-relativistic limit by construction.

With several configurations included it should in principle be possible to reach the correct non-relativistic limit, in practice one can, however, generally not achieve this in a truncated calculation. In practice the number of configurations has to be truncated for all but the smallest systems. It is common to truncate after double excita-
TABLE II: Contributions to the MCDF energy affected by the N.R. offset $(\mathrm{eV})$. " $\Delta E$ doub. Exc. $\rightarrow n=i$ " : correlation energy for the configuration space which include all double excitations up to principal quantum number $n=i$. Rel. Val.: Relativistic Value. N.R. Off.: Offset obtained at the nonrelativistic limit.

\begin{tabular}{|c|c|c|c|}
\hline & Rel. Val. & N.R. Off. & Diff. \\
\hline Dirac-Fock Coulomb & 2.31626 & -0.00148 & 2.31774 \\
\hline \multicolumn{4}{|c|}{ Brillouin single excitations excluded } \\
\hline$\Delta E$ Exc. $\rightarrow n=3$ & -0.01855 & -0.02086 & 0.00231 \\
\hline$\Delta E$ Exc. $\rightarrow n=4$ & -0.01421 & -0.01926 & 0.00505 \\
\hline$\Delta E$ Exc. $\rightarrow n=5$ & -0.01641 & -0.02247 & 0.00606 \\
\hline Total & 2.21621 & -0.02395 & 2.24016 \\
\hline Diff. With Exp. & -0.02389 & & 0.00006 \\
\hline \multicolumn{4}{|c|}{ All single and double excitations included } \\
\hline$\Delta E$ Exc. $\rightarrow n=3$ & -0.00371 & -0.00582 & 0.00211 \\
\hline$\Delta E$ Exc. $\rightarrow n=4$ & 0.00445 & -0.00037 & 0.00482 \\
\hline$\Delta E$ Exc. $\rightarrow n=5$ & 0.00661 & 0.00075 & 0.00586 \\
\hline Total (S.E.S. Welton) & 2.23923 & -0.00073 & 2.23996 \\
\hline Diff. With Exp. & -0.00087 & & -0.00014 \\
\hline
\end{tabular}

tions from the dominating configuration, but just as double excitations are needed to be added to the single excitations to obtain the correct non-relativistic limit, triple excitations will be needed to be added to corresponding double excitations and so on. Since higher multiple excitations are less important the remaining offset will however decrease steadily.

We now proceed to demonstrate the vanishing of the non-relativistis offset in an essentially complete MCDF calculation. In the present calculation we have added to the original configuration all single and double excitations up to a given maximum $n$ and $\ell$. Note that one has to be careful in considering the meaning of single and double excitations. For example the $1 s^{2} 2 s^{2} 2 p^{4} 3 p$ is a single excitation in the $L S$ coupling sense. Yet in $j j$ coupling it gives rise to 5 configurations in the $J=1 / 2$ case, two of which are double excitations in the $j j$ sense $\left(2 p_{1 / 2} 2 p_{3 / 2}^{4} \rightarrow 2 p_{1 / 2}^{2} 2 p_{3 / 2}^{2} 3 p_{1 / 2}\right.$ and $\left.2 p_{1 / 2}^{2} 2 p_{3 / 2}^{2} 3 p_{3 / 2}\right)$. We went from $3 d$ to $5 g$ for the case with a normal speed of light, and up to $6 h$ for the non-relativistic limit. This represents respectively $299,1569,4339$ and 9127 fully relaxed $j j$ configurations for the $J=1 / 2$ case, and 456 , 2541, 7356 and 15915 for the $J=3 / 2$. The calculations are repeated with different lists of configurations. In one group of calculations, we include all single and double excitation in the $j j$ sense, except for the "Brillouin single excitations", i.e., those that should contribute only in third order, as stated by Brillouin's theorem 9, 10, 11. These excitations are often excluded since they complicate the numerical convergence. Again we use here Brillouin's theorem in the $j j$ sense, i.e., we exclude all configurations transformed from the initial one by replacing 


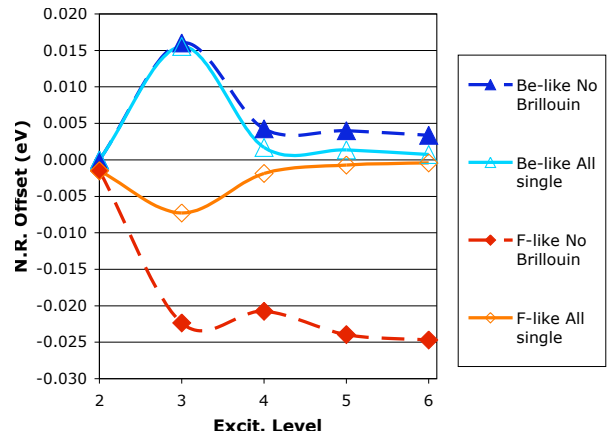

FIG. 2: Comparison of the non-relativistic offset for Be-like and F-like argon, evaluated including all single excitations, or only those not obeying Brillouin's theorem.

an orbital with quantum numbers $n, \kappa$ by one with $n^{\prime}, \kappa$, where $\kappa$ is the Dirac angular number. In a second group we include all single and double excitations. In both groups, we do calculations once with only the Coulomb interaction between electrons used in the evaluation of wavefunctions and energies, and once with the full Breit interaction in the evaluation of wavefunctions and mixing coefficients. This allows to include high-orders of the Breit interaction in the calculation. In each group the Coulomb only calculation is done also a second time with a large value for the speed of light. The evolution of the N.R. shift as a function of the maximum excitation used in the MCDF process is plotted in Fig. 2 for both Flike and Be-like ions, to show the generality of what is observed: the N.R. offset tends to a non-zero constant value when Brillouin configurations are excluded, and to zero when all single excitations are included.

The different contributions to the MCDF result, and the variation of the correlation energy and nonrelativistic offset with and without Brillouin configurations are presented in Table III When comparing both results, it is clear that excluding Brillouin single excitations and then subtracting the N.R. offset leads to the same result as including the Brillouin configurations. The agreement with experiment and with RMBPT results is excellent in both cases, even though the quality of the convergence when including all single excitations is not as good as when Brillouin ones are excluded. Moreover, the inclusion of all single excitations enables also to correct the energy of a level as shown in Table III which was not possible with the subtraction method. Finally we note that the evaluation of the radiative corrections, the self-energy screening (SES) with the help of the Welton approximation 12] leads to a very good agreement with experiment.

In conclusion we have proven, by comparing RMBPT and MCDF results, that the N.R. offset is due to relaxation and should go away when doing a complete calculation. We then showed that in the MCDF case, the offset is going to zero if a large enough configuration

TABLE III: Change in the ground state $(J=3 / 2)$ correlation energy due to Brillouin single excitation

\begin{tabular}{lrrr}
\hline \hline Conf. & No-brillouin & All single & Diff \\
\hline corr $\rightarrow 3 d$ & -5.1792 & -5.1989 & -0.0196 \\
corr $\rightarrow 4 f$ & -7.7349 & -7.7603 & -0.0255 \\
corr $\rightarrow 5 n$ & -86551 & -86871 & -0.0290
\end{tabular}

space is used, but only if all single configurations are included. In practice excluding Brillouin single excitations and then subtracting the N.R. offset leads to the same value, but numerical convergence of the self-consistent field process is much easier in the latter case. Finally, failing to account for the N.R. offset leads to poor results, even at a moderately large $Z$, a fact that may not have received enough attention in many MCDF calculations. The present work also shows that similar problems can happen in RMBPT calculations if subclasses of important effects are singled out and by themselves are treated to higher order. The improved convergence will then come at the expense of an N.R. offset. This fact had not been recognized before.

Laboratoire Kastler Brossel is Unité Mixte de Recherche du CNRS n ${ }^{\circ}$ 8552. Financial support for this research was received from the Swedish Science Research Councils (VR).

* Electronic address: paul@spectro.jussieu.fr

[1] P. Beiersdorfer, Annu. Rev. Astron. Astrophys. 41, 343 (2003).

[2] K. N. Huang, Y. K. Kim, K. T. Cheng, and J. P. Desclaux, Phys. Rev. Lett. 48, 1245 (1982).

[3] I. Draganic, J. R. Crespo López-Urrutia, R. DuBois, S. Fritzsche, V. M. Shabaev, R. Soria Orts, I. I. Tupitsyn, Y. Zou, , and J. Ullrich, Phys. Rev. Lett. 91, 183001 (2003).

[4] T. Mooney, E. Lindroth, P. Indelicato, E. G. Kessler, and R. D. Deslattes, Phys. Rev. A 45, 1531 (1992).

[5] P. Indelicato and E. Lindroth, Phys. Rev. A 46, 2426 (1992).

[6] P. J. Mohr and B. N. Taylor (2004), http://physics.nist.gov/cuu/Constants/index.html.

[7] P. J. Mohr and B. N. Taylor, Rev. Mod. Phys. 72, 351 (2000).

[8] J. A. Stone and J. H. Zimmerman (2004), http://emtoolbox.nist.gov/.

[9] J. Bauche and M. Klapisch, J. Phys. B: At. Mol. Phys. 5, 29 (1972).

[10] C. Froese Fischer, T. Brage, and P. Jönsson, Computational Atomic Structure (Institute of Physics Publishing, Bristol, 2000).

[11] M. Godefroid, J. Lievin, and J. Y. Metz, J. Phys. B: At. Mol. Phys. 20, 3283 (1987).

[12] P. Indelicato, O. Gorceix, and J. Desclaux, J. Phys. B: At. Mol. Opt. Phys. 20, 651 (1987). 